\title{
Electromagnetic Calorimetry for the ILC
}

\section{Cristina CÂRLOGANU*i}

Laboratoire de Physique Corpusculaire de Clermont-Ferrand, 24 avenue des Landais, 63177

Aubière CEDEX, France, IN2P3/CNRS, Université Blaise Pascal

E-mail: carlogan@in2p3.fr

The CALICE Collaboration builds electromagnetic and hadronic calorimeters optimised for particle flow at ILC. Two physics prototypes for the analog electromagnetic calorimeters (ScECAL and SiW ECAL) were extensively tested with beams. Both showed a remarkable facility of operation, low noise and high stability in time. The first analysis of the testbeam data indicate that they meet the ILC requirements in terms of linearity and single-particle energy resolution. The ScECAL shows a linearity within $6 \%$ for electrons from 1 to $32 \mathrm{GeV}$ and has a relative energy resolution of $(15.15 \pm 0.03$ (stat) $) / \sqrt{E(\mathrm{GeV})} \oplus(1.44 \pm 0.02$ (stat)) (\%). For electrons from 6 to $45 \mathrm{GeV}$, the SiW ECAL prototype is linear to within approximately the $1 \%$ level and has a relative energy resolution of $(16.53 \pm 0.14$ (stat) \pm 0.4 (syst) $) / \sqrt{E(\mathrm{GeV})} \oplus(1.07 \pm 0.07$ (stat) \pm 0.1 (syst) $)(\%)$.

European Physical Society Europhysics Conference on High Energy Physics, EPS-HEP 2009, July 16 - 222009

Krakow, Poland

* Speaker.

$\dagger$ On behalf of the CALICE collaboration 


\section{Highly Granular Calorimeters for the International Linear Collider}

The CALICE Collaboration is conducting R\&D into calorimetric systems for the ILC [1], a proposed $\mathrm{e}^{+} \mathrm{e}^{-}$linear collider intended to operate at a centre of mass energy ranging up to the $\mathrm{TeV}$ scale. Since the final states at ILC are typically multiple hadronic jets, their signature often relies on the identification of $\mathrm{Z}$ or/and $\mathrm{W}$ bosons in their decay modes into two jets. In order to distinguish them efficiently, a jet energy resolution close to $30 \% / \sqrt{E / \mathrm{GeV}}$ has to be achieved [1]. A precise reconstruction of the jet direction (few $\mathrm{mrad} / \sqrt{E / \mathrm{GeV}}$ ) is also required. The "particle flow" approach [2], which relies on the separate reconstruction of as many particles in the jet as possible, has the potential of achieving the required resolutions. The success of the algorithm heavily depends on the quality of the pattern recognition in the calorimeters, which means that a high spatial granularity for the calorimeters is as important as the intrinsic energy resolution for single particles.

The aim of the CALICE Collaboration is to build electromagnetic and hadronic calorimeters optimised for particle flow. In a first phase, the feasibility of building pixelised calorimeters is proven through the construction of "physics prototypes" which are operated and characterised in beam-tests. A subsequent, engineering-phase, is focused on the production of a "technological prototype", at the full ILC scale, which can be inserted and operated in a complete ILC detector. Several technologies are studied in parallel, both for the electromagnetic and hadronic calorimeters. Only the analog options for electromagnetic calorimetry will be presented here, since the developments for hadronic calorimetry were reported on in a different contribution at this conference [3].

The two CALICE analog options for the electromagnetic calorimetry are sampling calorimeters. In order to separate particles transversally, the absorber needs to have a small Molière radius. Conversely, both the need of compactness and of longitudinal separation require a small radiation length. The natural choice is tungsten, which in addition has a large interaction length to radiation length ratio. To contain high energy showers, the calorimeter requires $24 X_{0}$, distributed over 30 layers, such as to provide sufficient segmentation. Two choices are under study for the active material: plastic scintillator in the case of the ScECAL prototype and silicon in the case of the SiWECAL prototype. The two have high transversal granularity, $10 \times 10 \mathrm{~mm}^{2}$, and the non-active areas are as reduced as possible by integrating the electronics on the detector wherever possible.

\section{The ScECAL prototype}

The ScECAL [1] is proposed to realise a fine-segmented calorimetry in a stable, robust and cost-effective way. The effective $10 \times 10 \mathrm{~mm}^{2}$ lateral segmentation is obtained by using $1 \mathrm{~cm}$ wide and $4.5 \mathrm{~cm}$ long scintillator strips aligned orthogonally in adjacent layers. The scintillator layer thickness is $2 \mathrm{~mm}$, whereas the absorber thickness is of $3 \mathrm{~mm}$. The scintillator strips are cheaply produced by extrusion. The scintillation photons are collected by a wavelength shifting fibre, inserted centrally, along the length of each scintillator strip and read out with a Pixelated Photon Detector (PPD). The use of PPD's and highly integrated readout electronics leads to almost negligible dead area.

The first ScECAL prototype tested with beam had 26 scintillator layers interleaved with $3.5 \mathrm{~mm}$ thick tungsten-cobalt absorber layers, for a total of 18.5 interaction lengths. It was tested at DESY 
in March 2007 with positron beams from 1 to $6 \mathrm{GeV}$. The prototype was subsequently expanded (doubled in lateral size and four layers added) and improved by increasing the uniformity of the scintillator strips. This last prototype (Fig. 1, left) was tested at FNAL in September 2008 and May 2009 with electrons and positrons from 1 to $32 \mathrm{GeV}$ and pions from 1 to $60 \mathrm{GeV}$. The prototype showed a remarkable behaviour, with only 5 out of the 2160 channels not functioning. Each cell was individually calibrated with MIPs and the calibration constants are reasonably uniform over the detector (Fig. 1, right).

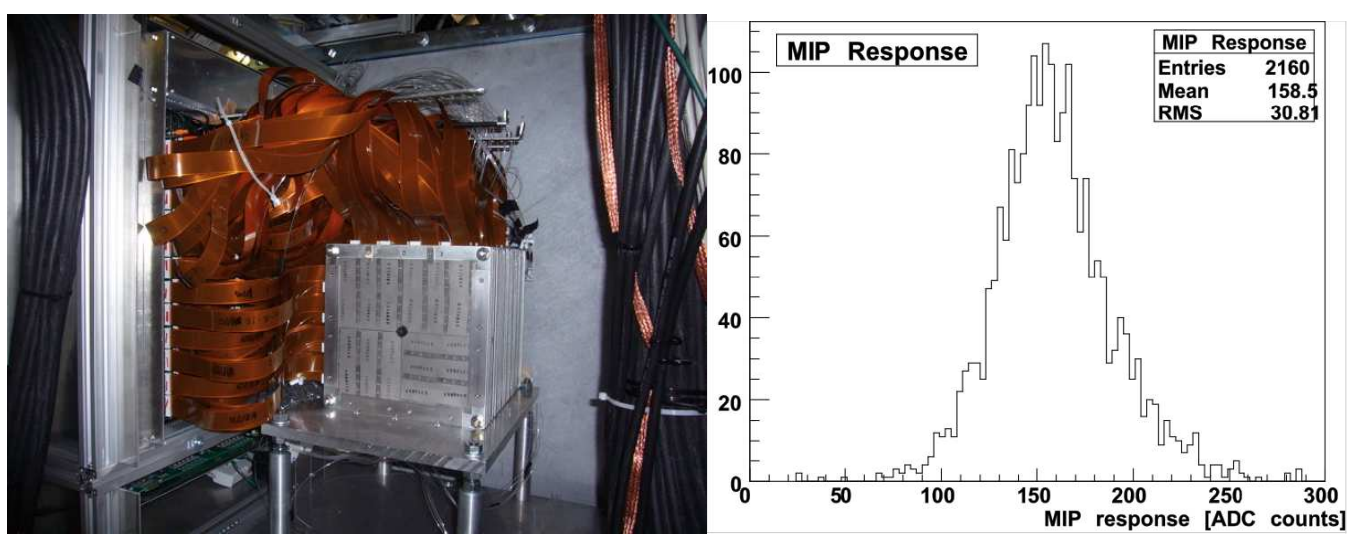

Figure 1: Left:The ScECAL prototype during the 2009 FNAL testbeam. Right: The distribution of the MIP calibration constants over the 2160 channels of the prototype.

The results from a preliminary analysis of the 2008 FNAL data indicate a linearity of the prototype response to electrons in the range from 1 to $32 \mathrm{GeV}$ better than $6 \%$. The relative energy resolution is estimated to be

$$
\frac{\sigma(E)}{E}=\left(\frac{15.15 \pm 0.03(\text { stat })}{\sqrt{E(\mathrm{GeV})}} \oplus(1.44 \pm 0.02(\text { stat }))\right) \%
$$

\section{The SiW ECAL prototype}

The silicon-tungsten option for the electromagnetic calorimeter [4] has high resistivity silicon as active material, which offers a large choice of diode size and therefore, of detector granularity and compactness. The tungsten modules, with an alveolar structure, allow the detection modules, called slabs, to slide in. Each slab has a tungsten layer sandwiched between two silicon active layers. To ensure a good energy resolution at low energy, the absorber thickness is varied longitudinally: the first ten layers have $0.4 X_{0}$ each, the next ten twice their thickness and the last ten $1.2 X_{0}$ each. The overall thickness of the prototype is $20 \mathrm{~cm}$. Each silicon layer has an active area of $18 \times 18 \mathrm{~cm}^{2}$, segmented into modules of $6 \times 6$ readout pads of $1 \times 1 \mathrm{~cm}^{2}$ each, leading to a total of 9720 channels for the whole prototype. The $525 \mu \mathrm{m}$ thick silicon-wafers are hold on 14-layer printed circuit boards, $2.1 \mathrm{~mm}$ thick and $600 \mathrm{~mm}$ long. A very-front-end ASIC was specifically designed to read out the silicon modules. The ASICs outputs are transmitted to the off-detector electronics using differential analogue lines. 
Since 2006, the SiW ECAL physics prototype was tested with electron, pion and muons beams at DESY, CERN and FNAL, over a large energy range. Blocks of random triggers were recorded during data-taking in order to monitor pedestals and noise. The noise level was typically $0.13 \mathrm{MIPs}$; its spread channel-to-channel was $9 \%$ of the mean noise and the spread run-to-run was less than $1 \%$ of the mean noise. Calibration constants for each pad were determined using muon events and the channel-to-channel dispersion was measured to be 5\%. At CERN, in 2006, all but 9 pads were functioning. Data taken in various beam-tests periods and locations showed well correlated calibration constants, with systematic shifts less than $2 \%$ of a MIP.

An example of energy recorded with the prototype for $30 \mathrm{GeV}$ electrons, during the 2006 testbeam at CERN is shown in Fig. 2, left, [5], together with a Gaussian fit and the expectation from simulation. As it can be seen in Fig. 2, right, where the residuals to linearity are shown, the prototype non-linearity is found to be within $1 \%$.

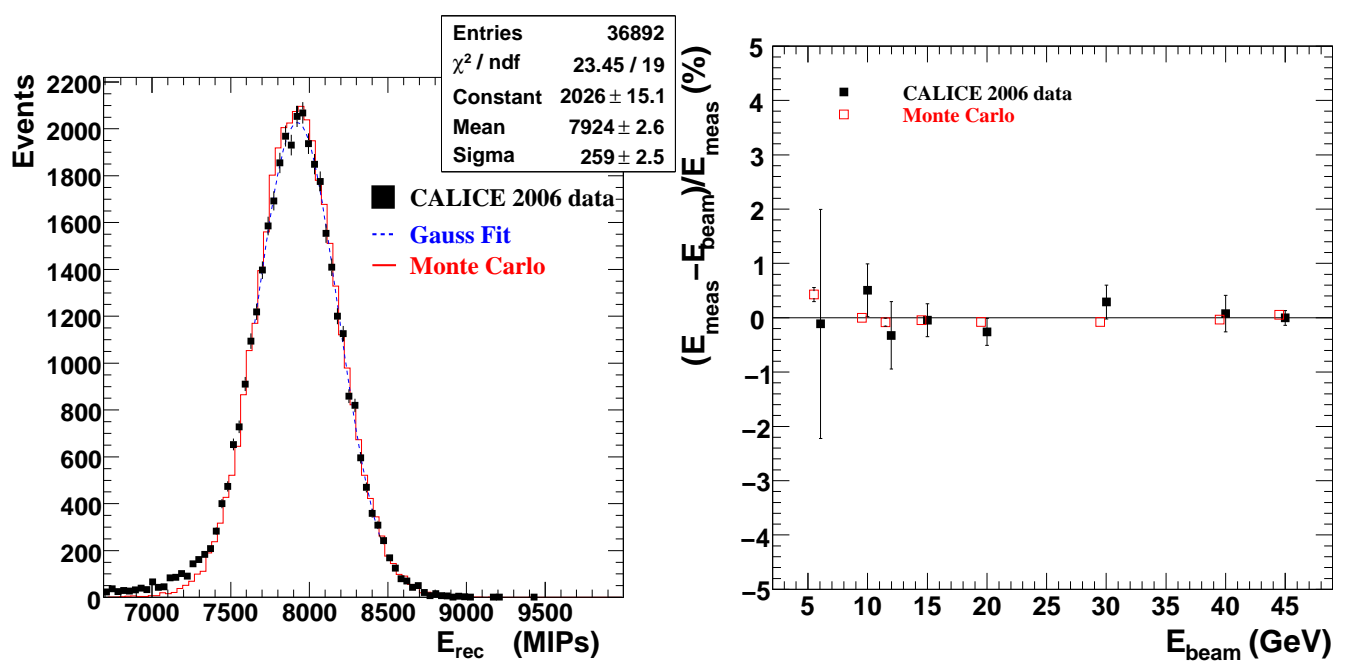

Figure 2: Left: Gaussian parametrisation of energy recorded in the prototype for $30 \mathrm{GeV}$ electron data (dashes). The data (solid squares) and simulation (continuous line) are superimposed. Right: The linearity of the response, $\left(E_{\text {meas }}-E_{\text {beam }}\right) / E_{\text {meas }}$, as a function of the beam energy, for data (solid squares) and simulation (open squares). For clarity, the Monte Carlo points were artificially shifted along the $x$ axis around the nominal $E_{\text {beam. }}$.

The relative energy resolution can be parametrised by a quadrature sum of stochastic and constant terms:

$$
\frac{\sigma(E)}{E}=\left(\frac{16.53 \pm 0.14(\text { stat }) \pm 0.4(\text { syst })}{\sqrt{E(\mathrm{GeV})}} \oplus(1.07 \pm 0.07(\text { stat }) \pm 0.1(\text { syst }))\right) \%
$$

The expected resolution from simulation agrees with the measured resolution of the prototype to within $2 \%$ of its value at all energies, except at $20 \mathrm{GeV}$, where the discrepancy is $3 \%$.

\section{Conclusion}

The tests with beams validated the CALICE physics prototypes. They showed that the necessary knowledge to build them was acquired: they are easy to operate, very stable in time, with 
low noise and robust and uniform MIP-calibrations. The first analysis of the testbeam data indicate that they meet the ILC requirements in terms of linearity and single-particle energy resolution. A reasonable agreement was reached between data and simulation. Analysis on shower development studies and tracking performance are ongoing.

The beam-tests showed also the way for some necessary improvements of the technology. They will be addressed, together with the engineering challenges linked to the integration of the calorimeters in the full ILC detector and the real-scale construction, with the technological prototypes currently under development.

\section{References}

[1] T. Behnke et al (ed.), Reference Design Report "Volume 4: Detectors", available at http: / / lcdev.kek.jp/RDR

[2] D. Buskulic et al, "Performance of the ALEPH detector at LEP", Nucl.Instrum.Meth.A360:481-506,1995.

[3] Jaroslav Cvach, "The CALICE hadron calorimeters - test beam results”, these proceedings.

[4] M. Anduze et al, "Design and Commissioning of the Physics Prototype of a Si-W Electromagnetic Calorimeter for the International Linear Collider", JINST 3 (2008) P08001, 33p.

[5] C. Adloff et al, "Response of the CALICE Si-W Electromagnetic Calorimeter Physics Prototype to Electrons”, NIM A608 (2009) 372. 